Association for Information Systems

AIS Electronic Library (AISeL)

\title{
A Methodology to enhance Learning Processes with Augmented Reality Glasses
}

Tobias Dreesbach

German Research Center for Artificial Intelligence (DFKI), Smart Enterprise Engineering, Osnabrück, Germany

Matthias Berg

Saarland University, Forschungsinstitut Bildung Digital (FoBiD), Saarbrücken, Germany

Henning Gösling

German Research Center for Artificial Intelligence (DFKI), Smart Enterprise Engineering, Osnabrück, Germany

Tobias Walter

Saarland University, Forschungsinstitut Bildung Digital (FoBiD), Saarbrücken, Germany

Oliver Thomas

German Research Center for Artificial Intelligence (DFKI), Smart Enterprise Engineering, Osnabrück, Germany

See next page for additional authors

Follow this and additional works at: https://aisel.aisnet.org/wi2021

Dreesbach, Tobias; Berg, Matthias; Gösling, Henning; Walter, Tobias; Thomas, Oliver; and Knopf, Julia, "A Methodology to enhance Learning Processes with Augmented Reality Glasses" (2021).

Wirtschaftsinformatik 2021 Proceedings. 3.

https://aisel.aisnet.org/wi2021/KDigitalEducation15/Track15/3

This material is brought to you by the Wirtschaftsinformatik at AIS Electronic Library (AISeL). It has been accepted for inclusion in Wirtschaftsinformatik 2021 Proceedings by an authorized administrator of AIS Electronic Library (AISeL). For more information, please contact elibrary@aisnet.org. 


\section{Presenter Information}

Tobias Dreesbach, Matthias Berg, Henning Gösling, Tobias Walter, Oliver Thomas, and Julia Knopf 


\title{
A Methodology to enhance Learning Processes with Augmented Reality Glasses
}

\author{
Tobias Dreesbach ${ }^{1}$, Matthias Berg ${ }^{2}$, Henning Gösling ${ }^{1}$, Tobias Walter ${ }^{2}$, Oliver \\ Thomas $^{1}$, and Julia Knopf ${ }^{2}$ \\ ${ }^{1}$ German Research Center for Artificial Intelligence (DFKI), Smart Enterprise Engineering, \\ Osnabrück, Germany \\ \{tobias.dreesbach,henning.goesling,oliver.thomas \}@dfki.de \\ ${ }^{2}$ Saarland University, Forschungsinstitut Bildung Digital (FoBiD), Saarbrücken, Germany \\ \{matthias.berg,tobias.walter,julia.knopf\}@fobid.org
}

\begin{abstract}
Although Augmented Reality (AR) encourages self-managed learning and enhances the learner's reflection only few companies and schools use AR glasses in vocational training. In our research project we aim to support the dissemination of AR and speed up the augmentation of existing learning processes. But we did not come across methodologies for transferring a conventional learning processes into learning processes for $\mathrm{AR}$ Glasses. Therefore, we developed a methodology to enhance conventional learning processes with AR glasses by supporting the creation of AR enrichments for the individual process steps. We describe a use case of an electro engineering lesson that is enriched with AR elements using our methodology.
\end{abstract}

Keywords: Augmented Reality Glasses, Learning Process, Methodology

\section{$1 \quad$ Introduction}

With Augmented Reality (AR), virtual 3D objects are integrated into a real environment in real time [1]. AR learning experiences enhance learning gains as well as motivation and help students to perform learning activities [2-3]. AR glasses capture the environment and integrate virtual elements in the user's field of view. Areas of application include a wide range of topics, target groups and academic levels [4]. Several research projects target the field of AR in vocational training, e.g. [5-6]. AR in vocational training encourages self-managed learning for direct instructions and enhances the reflection of the learners in task-oriented settings [7]. However, just a small number of the surveyed companies in a recent study use AR glasses in their vocational training [8]. Main concerns according to [9] are social consequences, privacy, security and a missing added value. To spread the use of AR in vocational training, we propose a methodology for the conversion of conventional learning processes into AR-based learning processes that takes the technical limitations of AR glasses into account. More precisely, with our methodology we want to ease and speed up the conversion of learning processes to AR-based learning processes. We aim to

16th International Conference on Wirtschaftsinformatik,

March 2021, Essen, Germany 
encourage the didactical use of AR glasses in learning settings so that more students can experience AR glasses with their potentials [7]. Beyond that our research provides suggestions to people who are not familiar with the implementation of AR applications. A few methodologies for the integration of AR devices in specific domains can already be found in the literature. For example, for the integration of AR into an intralogistics context [10] and into an Industry 4.0 context [11]. In the field of education, [12] proposed a methodology consisting of three steps to integrate AR devices into practical learning processes in an industrial environment: (1) A process analysis using the Business Process Model and Notation (BPMN) as the modelling language for the documentation of the conventional learning process. (2) Definition of process types and their AR potential. (3) Identification of the suitable AR device (tablet, smartphone, smart glasses) for each process type and implementation of an application for the ARbased training. A methodology for how to use AR glasses to improve the vocational training is given by [13]. They provide a list of design elements to exploit AR and wearable sensors for training purposes, e.g. with augmented paths to guide the trainee's motions or interactive virtual objects. For some of the design elements the expert's performance is captured in a certain process step with sensors and the trainees use AR glasses to project the captured data into their field of view. As we did not come across an established methodology to enhance existing conventional learning processes with AR glasses, we examined our research with the following research question: Which steps are necessary to enhance a conventional learning process with AR glasses?

In our ongoing research project, we evaluate the methodology regarding four technical use cases - one of those from a metalworking company we present in this paper.

\section{Development of the Methodology}

In our research project we identified the problem of a missing structured approach to convert conventional learning processes into AR-based processes. Therefore we used the design science research methodology (DSRM) proposed by [14] to enhance learning processes with AR glasses by supporting the creation of AR enrichments for the individual process steps. We used the DSRM, because it provides well-established guidelines for research in information systems and helps us to insure the relevance and effectiveness of our research output. The DSRM includes six steps, as shown in figure 1. We identified the problem (step 1) when we documented four processes in a technical training with BPMN to convert them to AR-based learning processes: (1) the operation of a milling machine, (2) the assembly of a two-way circuit, (3) an air conditioner, and (4) the programming of a servomotor. We decided to (step 2) develop a methodology for creating and adapting AR elements to the individual process steps after we scrutinized the BPMN models. In a literature review we identified applicable methodologies for the integration of AR devices in educational processes. For the design and development (step 3), we interviewed nine teachers and trainers in the field of vocational training about the didactic theory behind the use cases. We created a didactical and technical concept out of the results and applied the concepts to the learning processes. We are now implementing a prototype for AR glasses (step 4) 
covering the elicited use cases. During the evaluation (step 5), we will iterate back to compare our initial objectives to the actual observed results and to improve the effectiveness of our knowledge contribution [14]. We will evaluate our methodology by conducting a summative test [15] where we will measure the effects of the AR-based learning processes. A case study with students will be performed as well as expert interviews with teachers and trainers.

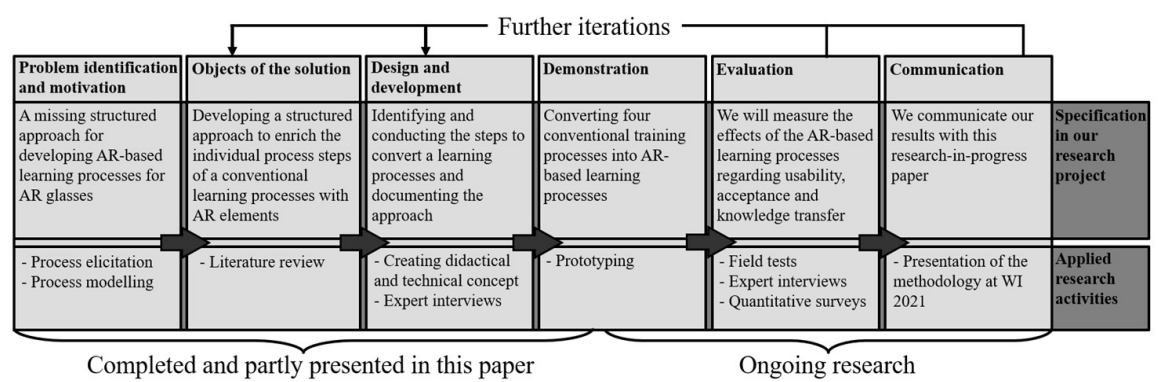

Figure 1. Applied design science research method adopted from [14]

We divided our methodology in five consecutive steps:

1. Process analysis. An analysis of the problem statement is recommended. The inspection of the actual process and its environment provides an uniform understanding of the process [10]. Involved participants depend on the learning process and can have multiple roles which are the process owners (like teachers, trainers, foremen), didactic experts, students, trainees, AR experts, developers, and a project manager. The process owners make suggestions for the process selection and explain the process activities. All teaching materials are reviewed by the participants, and a shadowing approach is used during a learning session to observe possible failures and deviations regarding the optimal process. A process model is prepared to document the recorded process and its subprocesses. We suggest BPMN as the modelling language similar to [10] and [12]. Finally, the documented BPMN process is discussed with the process owners to confirm the correctness and completeness.

2. AR element selection. If the BPMN model is complete and consistent, all process steps in the BPMN model are evaluated regarding their potential for an AR enrichment. AR elements can be bound to the world to stay at the same position as the user moves around. They can also be bound to the user's device so that they are persistently available but not in a distracting manner. A wide range of AR elements to enrich a process is already discussed in the literature. Some examples for generic elements are: pictures, videos, explosion diagrams, X-ray visuals to uncover hidden structures and assisting visual aids like arrows as well as guideline and highlight elements [16]. For a wide range of AR elements [13] present how to use them for industrial training. Sometimes markers are needed to anchor the position of virtual objects to the real world. Moreover, a combination of the AR elements can increase the context sensitivity. While the AR experts present suggestions for possible AR elements, the process owners and didactic experts including the teachers and trainers express their 
demands and evaluate the AR element's didactical value for the individual process steps. The role of the developers is to give feedback regarding the technical feasibility based on a shortlist of AR devices. If all participants agree to the selection, the project manager adds the AR elements as comments to the BPMN process steps. When the elements are not sufficient to support the process step new elements must be created.

3. Creation of new AR elements. When the process requires the development of new AR elements, the need of improvement for a process step and the underlying didactical concept is discussed between all participants and requirements are defined. Ideas for suitable realizations can also be suggested from all participants. In consideration of the didactical concept, the AR experts and the developers propose technical feasible and context-sensitive AR elements to enrich the process step and get feedback for it. If possible, a generic structure of the AR elements is preferred to reduce the effort. Generic elements can be reused with new content and combined with other AR elements in further process steps.

4. Clarification of the process concept. If all process steps are enriched, the project manager transfers the BPMN model with the AR enrichments into a detailed process concept which is provided to all participants. The concept contains a list of all process steps and their AR enrichment. It documents the demands of all participants and summarizes the results of the technical feasibility. A visual mock-up for the AR elements can be added to the concept.

5. Effort and feasibility review. In the last stage of our methodology the results are evaluated in a group discussion between all project participants. If problems in the concept are detected or demands are not fulfilled the process concept is adapted. The $\mathrm{AR}$ experts and developers select $\mathrm{AR}$ glasses based on the requirements and the predefined shortlist. After the successful evaluation, the implementation begins.

\section{Methodology Application for a Technical Learning Session}

In one of our use cases, recorded in a metalworking company, basic knowledge and skills in the field of electrical engineering are imparted during the construction of a multiway switching with one light bulb and two light switches. First, the trainees receive an instruction and theoretical background in the field of electrical engineering and must draw a circuit diagram. In a following practical part, they assemble the circuit based on their plan and check its functionality on a test stand. The assembly sequence is not prescribed. By acting independently in an authentic situation, new knowledge structures are supported which, according to the principles of situated learning, prevent inert knowledge [17]. For the application of our methodology we documented the process using a BPMN model, reviewed all teaching materials, and used a shadowing approach during a session to observe deviations regarding the optimal process. Since our documented BPMN process was discussed with the process owners we continued with the second step of our methodology. The AR experts of our project suggested AR enrichments for most steps of the process model by combining AR elements that were known from the literature and presented it to the process owners and didactical experts. The AR elements included text elements, pictures, and videos for the explanation of the 
process steps, checklists to support the self-management of the students and trainees, buttons to select working tasks and confirm their completion, a progress bar, a timer, and three-dimensional arrows to highlight physical artifacts within the physical location. QR codes were suggested to anchor the AR elements within a workplace like in other AR applications [18]. In the following discussion among all participants of our multidisciplinary team we worked out that the AR elements are sufficient for most process steps but additionally individual AR elements are required to support situated learning scenarios in the respective learning occupations - basically when a step was interactive, context-sensitive, collaborative, or strongly domain specific. In the third methodology step the required individual elements were developed. A challenging task was to check the accurate setup of the electrical parts and the wiring because the AR devices cannot interpret human actions during the assembly. The participants discussed the technical limitations of object detection and pattern recognition and, as a result, the process steps were enhanced with multiple choice queries, drag-and-drop matching tasks, and a questionnaire for the self-reflection if a task was successfully performed. During our fourth methodology step the enriched process was continuously updated in our process concept and reviewed by the process owners. We suggest adding value to the process concept by using mock-ups, which, in our use case, helped all participants to achieve a common understanding of the AR elements. A positive indicator of our methodology is that in the last step, the effort and feasibility review, no serious complications were detected.

\section{Conclusion and Outlook}

In this paper we present a methodology to enhance conventional learning processes with AR glasses by supporting the creation of AR enrichments for the individual process steps. Our methodology is based on five steps: process analysis, AR element selection, creation of new AR elements, clarification of the process concept, and effort and feasibility review. We present the application of the methodology for a learning process in an electrical engineering lesson. Due to the domain of our four use cases the methodology is so far limited to vocational training processes. In the current section of our research project we are implementing the AR elements for the use cases. We are in the first iteration of the DSRM and the evaluation of our implemented AR elements will start soon by measuring the didactical effects of our AR-based learning processes. A case study will be conducted to test the effectiveness of our methodology where two groups of trainees perform the learning process either with or without the AR Glasses while we measure their learning outcomes. Moreover, we will enrich more learning processes with our project partners to validate the efficiency of our methodology.

\section{Acknowledgements}

This Paper is accrued as part of the research project AdEPT (FKZ: 01PV18008E). The project is fostered from Federal Ministry of Education and Research (BMBF). We thank for the support enabled through that project. 


\section{References}

1. Azuma, R.T.: A Survey of Augmented Reality. Teleoperators Virtual Environ. 6, 355385 (1997)

2. Thomas, O., Metzger, D., Niegemann, H..: Digitalisierung in der Aus- und Weiterbildung. Springer Gabler, Berlin (2018)

3. Ibáñez, M.-B., Delgado-Kloos, C.: Augmented reality for STEM learning: A systematic review. Comput. Educ. 123, 109-123 (2018)

4. Garzón, J., Pavón, J., Baldiris, S.: Systematic review and meta-analysis of augmented reality in educational settings. Virtual Real. 23, 447-459 (2019)

5. Limbu, B.: WEKIT Framework \& Training Methodology. Technical report (2019)

6. Utzig, S., Kaps, R., Azeem, S.M., Gerndt, A.: Augmented Reality for Remote Collaboration in Aircraft Maintenance Tasks. IEEE Aerospace Conference, 1-10 (2019)

7. Lester, S., Hofmann, J.: Some pedagogical observations on using augmented reality in a vocational practicum. Br. J. Educ. Technol. 51, 645-656 (2020)

8. F. Siepmann, Fleig, M.: eLearning BENCHMARING Studie 2020. Siepmann Media, Hagen im Bremischen (2020).

9. Harborth, D.: Unfolding Concerns about Augmented Reality Technologies: A Qualitative Analysis of User Perceptions. Wirtschaftsinformatik, 1262-1276 (2019)

10. Berkemeier, L., Zobel, B., Werning, S., Ickerott, I., Thomas, O.: Engineering of Augmented Reality-Based Information Systems: Design and Implementation for Intralogistics Services. Bus. Inf. Syst. Eng. 61, 67-89 (2019)

11. Gattullo, M., Scurati, G.W., Fiorentino, M., Uva, A.E., Ferrise, F., Bordegoni, M.: Towards augmented reality manuals for industry 4.0: A methodology. Robot. Comput. Integr. Manuf. 56, 276-286 (2019)

12. Sorko, S.R., Brunnhofer, M.: Potentials of Augmented Reality in Training. Procedia Manuf. 31, 85-90 (2019)

13. Limbu, B., Fominykh, M., Klemke, R., Specht, M., Wild, F.: Supporting Training of Expertise with Wearable Technologies: The WEKIT Reference Framework. In: Yu, S., Ally, M., Tsinakos, A. (eds.) Mobile and Ubiquitous Learning, pp. 157-175. Springer, Singapore (2018)

14. Peffers, K., Tuunanen, T., Rothenberger, M.A., Chatterjee, S.: A design science research methodology for information systems research. J. Manag. Inf. Syst. 24, 45-77 (2007)

15. Gregor, S., Hevner, A.R.: Positioning and Presenting Design Science Research for Maximum Impact. MIS Q. 37, 337-355 (2013)

16. Keil, J., Schmitt, F., Engelke, T., Graf, H., Manuel, O.: Augmented reality views: Discussing the utility of visual elements by mediation means in industrial AR from a design perspective. In: Int. Conf. on Virtual, Augmented and Mixed Reality (pp. 298312). Springer, Cham (2018)

17. Mandl, H., Kopp, B., Dvorak, S.: Aktuelle theoretische Ansätze und empirische Befunde im Bereich der Lehr-Lern-Forschung - Schwerpunkt Erwachsenenbildung -. Technical Report, Deutsches Institut für Erwachsenenbildung (2004)

18. Bacca, J., Baldiris, S., Fabregat, R., Graf, S., Kinshuk: International Forum of Educational Technology \& Society Augmented Reality Trends in Education: A Systematic Review of Research and Applications. Educ. Technol. 17, 133-149 (2014) 